\title{
Influence of nutrient factors on phosphorus accumulating organisms (PAOs) Bacillus sp. PK1
}

\author{
Qian Zhang, Yao Li, Chunbiao Li, Hui Ku, Meng Li, Chenhui Jia, and Ziyi Xu
}

School of Civil Engineering and Architecture, Wuhan University of Technology, Wuhan 430070, China

vicki8346@163.com

\begin{abstract}
Keywords: phosphorus accumulating organisms (PAOs); Bacillus sp. PK1; initial phosphorus; Mixed carbon sources; phosphorus removal

Abstract: A kind of phosphorus accumulating organism (PAO), Bacillus sp. PK1, which showed high phosphorus removal efficiencies, was isolated from special medium in the SBR reactor. To further explore the effect of nutrient factors on its potential phosphorus removal efficiency, initial phosphorus content and various carbon sources were selected in this research. Results of a series tests showed that the initial phosphorus removal efficiency declined constantly to below $60 \%$ with the initial phosphorus content increased. As for the effect of carbon sources, the phosphorus removal efficiencies were respectively as high as $98.58 \%, 95.14 \%$ and $98.45 \%$ with sodium acetate, sodium citrate and mixed carbon source. More importantly, research also showed an infrequent phenomenon that the PK1 gave priority to utilize sodium citrate rather than short chain fatty acid sodium acetate, and hardly to use glucose.
\end{abstract}

\section{Introduction}

The phosphorus is one of the main factors which will cause eutrophication. A large amount of sewage, which does not reach the corresponding discharge standard, from industrial and agricultural production enters the nature water environment should be responsible for this. Hence, controlling and reducing the content of phosphorus in the treated sewage is one of the main tasks to prevent eutrophication. Phosphorus removal technologies mainly include physical, chemical, biological methods and combination of them above so far [1]. However, physical method is very rarely used because of its high cost and complex technology. And dealing with chemical method not only needs higher cost but also needs dosing chemical agent which will cause secondary pollution to the environment with a large amount of sludge, although it can achieve higher and stable phosphorus removal effect. In order to overcome these disadvantages above, a promising approach named biological phosphorus removal was reported 1950s. The biological phosphorus removal includes two categories: one absorbs phosphorus by biological assimilation (such as artificial wetland) and the other, which is called enhanced biological phosphorus removal (EBPR), is to utilize functional bacterium to over-uptake phosphorus [2]. Since EBPR has economic and environmental sustainable advantage, its mechanism and application become a hot research spot across the world recently.

In recent years, several studies found that the EBPR maybe could not be confined to the traditional anaerobic/aerobic alternately [3]. These researches show that microorganisms in different environmental conditions (such as the single aerobic condition) can also realize the excessive intake of phosphorus. A large number of experiments and engineering practices have proved that functional microbes (such as phosphorus accumulating organisms, PAOs) play a key role in biological phosphorus removal [4]. Nevertheless, there are still little type of pure breed efficient phosphorus removal bacteria which has been isolated so that there has even less study about their characteristics and functional mechanism. In addition, research on the utilization and metabolism of the ingredients of mixed carbon source is rare [5].

Thus, the purpose of this research was to explore the effect of nutrient factors (trace element, phosphorus consent and various carbon sources including sodium acetate, sodium citrate, glucose along with mixed carbon sources) on the potential phosphorus removal efficiencies of a new kind of 
phosphorus removal bacteria, Bacillus sp. PK1. It has great significance to promote the competitiveness of PK1 on the practical engineering application.

\section{Materials and methods}

\section{Source of strain}

The strains PK1, which were identified as Bacillus sp. based on its physiological and biochemical characters and 16S rDNA sequence, were isolated from the active sludge of anaerobic/aerobic/anoxic SBR reactor which had been operated stably in laboratory. For each test, the strain PK1 was treated with pregrown: First, controlling suitable conditions was completed for enlarging cultivation. Second, creating an anaerobic environment was to look towards fully phosphorus release of strain PK1. Finally, centrifugal washing processing was made.

\section{Initial phosphorus concentration test}

In addition to altering the initial phosphorus concentration $(8,12,16$ and $20 \mathrm{mg} / \mathrm{L})$ rather than the dosage of trace elements, the influences of initial phosphorus concentration were conducted under the same experimental conditions with trace elements test. Similarly, the concentration of $\mathrm{PO}_{4}{ }^{3-}-\mathrm{P}$ and DW was monitored.

\section{Carbon source tests}

\section{Single carbon source}

Inoculating strain PK1 in the phosphorus uptake medium [6,7] and then cultivated in an OCXO $\left(30^{\circ} \mathrm{C}, 120 \mathrm{rpm} / \mathrm{min}\right)$. Changing the carbon source of phosphorus uptake medium and controlling the molar volume of carbon at the same level when different single carbon source (sodium acetate, sodium citrate and glucose) was used. And the dosage of those three carbon sources respectively were: sodium acetate, $3.23 \mathrm{~g} / \mathrm{L}$; sodium citrate, $2.33 \mathrm{~g} / \mathrm{L}$; glucose, $1.57 \mathrm{~g} / \mathrm{L}$. And the liquid was sampled every $3 \mathrm{~h}$ for $24 \mathrm{~h}$. Then, the concentration of $\mathrm{PO}_{4}{ }^{3-}-\mathrm{P}, \mathrm{COD}_{\mathrm{cr}}$ and $\mathrm{DW}$ was monitored.

\section{Mixed carbon source}

Replacing the carbon source of phosphorus uptake medium with mixed carbon source, which consisted of sodium acetate $(1.0767 \mathrm{~g} / \mathrm{L})$, sodium citrate $(0.7767 \mathrm{~g} / \mathrm{L})$ and glucose $(0.5233 / \mathrm{L})$ according to same molar mass. The whole COD content was $1552 \mathrm{mg} / \mathrm{L}$. And then, the test was carried out in the same method with single carbon source. Besides $\mathrm{PO}_{4}{ }^{3-}-\mathrm{P}$ and $\mathrm{COD}_{\mathrm{cr}}$, the concentration variation of the three carbon sources was also measured.

\section{Chemical analysis}

Those above-mentioned indexes were measured in accordance with Standard Methods for the Examination of Water and Wastewater, 2012 [8]. And the compositional variation of sodium acetate and sodium citrate were assayed with high performance liquid chromatography (HPLC) method. The specific operating conditions of HPLC (Shimadzu, Japan) were as follows: carbon column TC-C18, column temperature $25^{\circ} \mathrm{C}$, wavelength $215 \mathrm{~nm}$, mobile phase methanol (5\%) and trifluoroacetic acid (TFA, $4.5 \%$ ), flow rate $1.0 \mathrm{~mL} / \mathrm{min}$, sample amount $10 \mu \mathrm{L}$ and diode array detector (DAD). 


\section{Results and Discussions}

\section{Influence of initial phosphorus concentration}

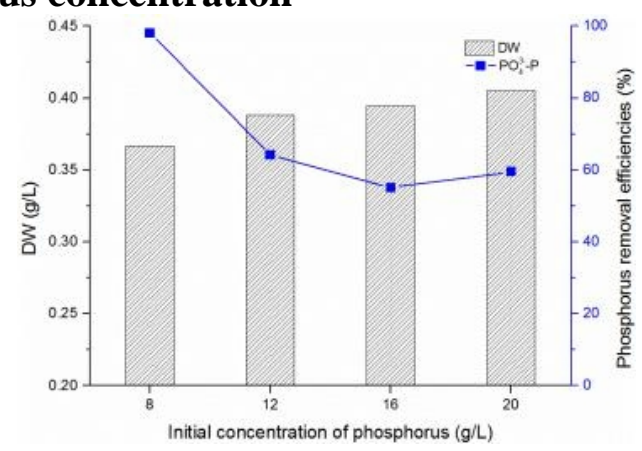

Fig. 1 Impact of initial phosphorus concentration

The biomass growth and phosphorus removal efficiency under different initial phosphorus levels were shown in Fig. 1. The phosphorus removal efficiency reached the maximum value $(98.05 \%)$ when the initial concentration of phosphorus was $8 \mathrm{~g} / \mathrm{L}$. However, it declined rapidly to a relatively stable level ( $60 \%$ or so) with the increase of initial phosphorus concentration while the change trend of DW was opposite. The DW increased slowly from $0.3667 \mathrm{~g} / \mathrm{L}$ to $0.4052 \mathrm{~g} / \mathrm{L}$.

It could be known that the total amount of phosphorus removal increased continuously, although the removal rate decreased continuously. It was deduced that the increase of initial phosphorus concentration was not only conducive to the growth of PK1 but also could stimulate the activity of phosphorus acid induced enzyme so that the total phosphorus uptake was improved. Therefore, the visually result was that the phosphorus removal rate did not increased but appeared a downward trend with the increase of initial phosphorus concentration.

\section{Influence of carbon sources}

\section{Single carbon source}
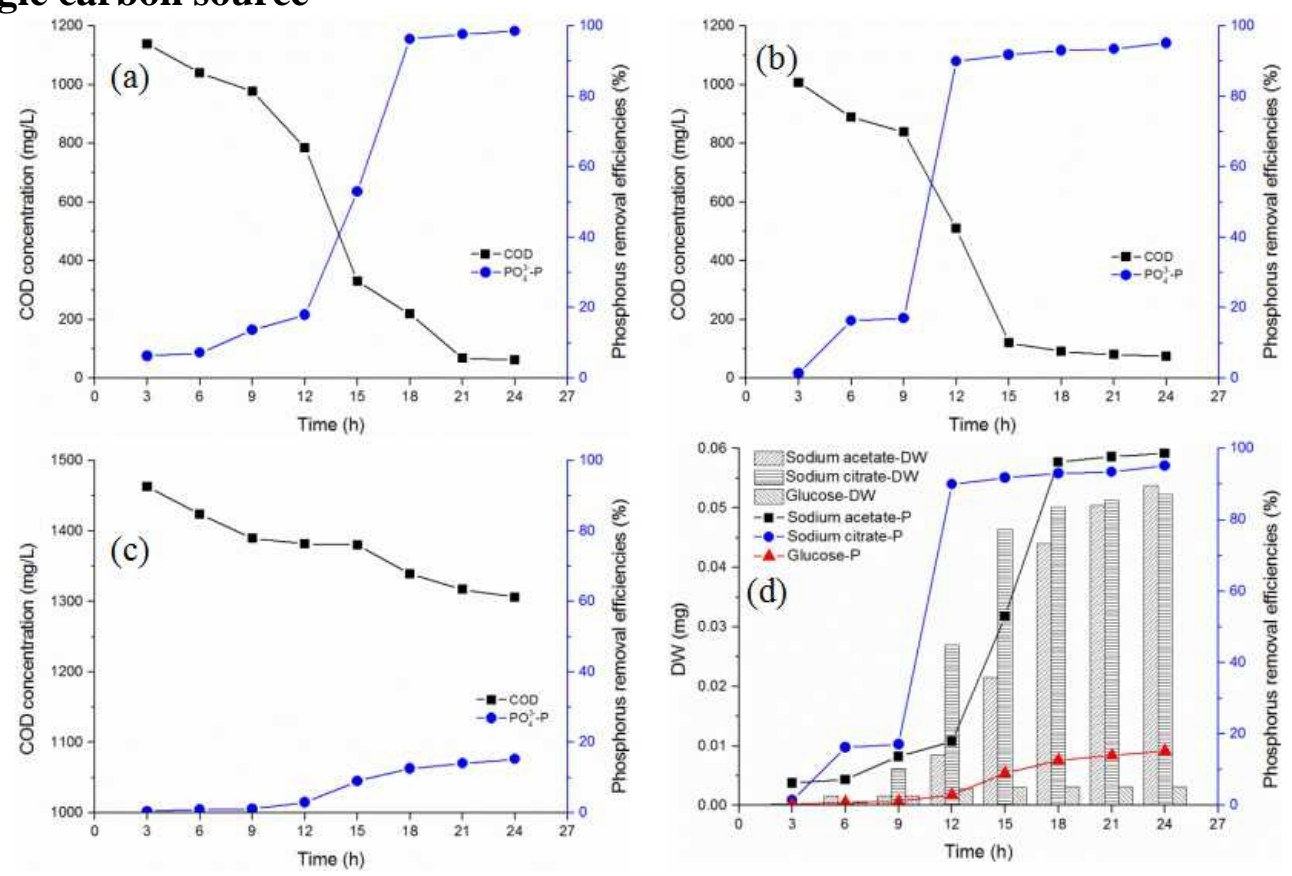

Fig.2 Impact of (a) sodium acetate, (b) sodium citrate,

(c) glucose and (d) three different carbon sources

It could be seen from Fig. 2(a) that the removal efficiency of $\mathrm{PO}_{4}{ }^{3-}-\mathrm{P}$ showed slow increasing in the initial $12 \mathrm{~h}$. But after $12 \mathrm{~h}$, the removal efficiency showed rapidly increase until $18 \mathrm{~h}$ with the corresponding maximum removal efficiency $96.21 \%$. And then the increasing trend became relatively smooth till $98.58 \%$ in the remaining 6 h. Sodium acetate, a kind of volatile short chain fatty acid, seemed to be easily absorbed via an active transport and stored as PHB by PK1. Therefore, the rapid 
degradation of COD was presented. Furthermore, taking use of the energy of PHB, PK1 could compound self cells and maintain life activities, and on the other hand, could intake the inorganic phosphate from the external environment and stored in the bacteria PK1 as poly-P [9].

Using sodium citrate as the carbon source, the removal rate of $\mathrm{PO}_{4}{ }^{3-}-\mathrm{P}$ reached more than $90 \%$ within $12 \mathrm{~h}$ and reached $95.14 \%$ eventually (Fig. 2(b)). Comparing sodium citrate with sodium acetate, the changing trends of the $\mathrm{PO}_{4}{ }^{3-}-\mathrm{P}$ removal rate and CODcr concentration were similar. It was a remarkable fact that both the phosphorus removal and COD concentration showed significantly changes during the period of 9-12h. This illustrated that PK1 could quickly absorb sodium citrate and stored in the form of PHB and PHV to absorb inorganic phosphate and further lead to the increase of the degradation of COD and phosphorus removal rate.

It had been shown in Fig. 2(c) that $\mathrm{PO}_{4}{ }^{3-}-\mathrm{P}$ removal efficiency was low with the maximum value $15.28 \%$ during the whole sampling period. The change of COD was also not obvious. By the above results, it could be deduced that glucose was not beneficial to the $\mathrm{PO}_{4}{ }^{3-}-\mathrm{P}$ removal in this experiment. It could be explained that the metabolism of glucose was complex. Some researches had shown that glucose was an involatile short chain fatty acids $[10,11]$. The glucose must be broken down into small molecule organic matter firstly before it could be absorbed by the PAOs. Thus it reduced the utilization efficiency of denitrifying phosphorus removal bacteria PK1 to glucose, which would further influence the $\mathrm{PO}_{4}{ }^{3-}-\mathrm{P}$ and $\mathrm{COD}$ removal efficiency. On the other hand, Carucci [12] and Cech [13] have reported that glucose would replace the intracellular glycogen to provide energy and reduce the biological growth; therefore, it promoted the growth of GAOs selectively and reduced the competitiveness of PAOs, thus affecting the absorption of phosphorus.

Fig. 2(d) intuitively illustrates that different carbon sources demonstrated distinct behaviors on phosphorus removal and the growth of PK1. The phosphorus removal rates both could reach more than $90 \% 18 \mathrm{~h}$ later with sodium acetate and sodium citrate. Meanwhile, the DW all reached up to $0.35 \mathrm{mg} / \mathrm{L}$ at the end. However, the phosphorus removal rate just increased to $15.3 \%$ and the dry weight was less than $0.05 \mathrm{mg} / \mathrm{L}$ accordingly when using glucose as carbon source alone. Furthermore, sodium citrate seemed to be easier to be utilized by PK1 than sodium acetate especially during the period of $9-12 \mathrm{~h}$. During this period, the removal rate with sodium citrate increased rapidly from $17.1 \%$ to $90.0 \%$, whereas it increased merely from $13.7 \%$ to $18.0 \%$ with sodium acetate. Furthermore, it could also found that PK1 absorbed COD and phosphorus in a certain proportion of 180 in stable state, which reflected the strong ability of PK1 to absorb phosphorus.

Summarizing the experimental results, it could be deduced that the phosphorus removal of strain PK1 mainly occurred in logarithmic phase and stable phase. And maybe the specific reasons for the uncommon phenomenon that strain PK1 was more likely to utilize sodium citrate rather than sodium acetate could be explained as follows. Compared with sodium acetate, sodium citrate could be directly involved in the tricarboxylic acid cycle (TAC) so that it could be adsorbed by strain PK1 more rapidly, whereas the utilization pathway of sodium acetate was relatively complicated. Some studies have found that when microorganism growth in substrate with acetic acid as the sole carbon source, it needs participated in glyoxylate cycle [14-16]. And then the product of glyoxylate cycle would turn into the TCA and continue to participate in metabolism $[17,18]$.

Mixed carbon source
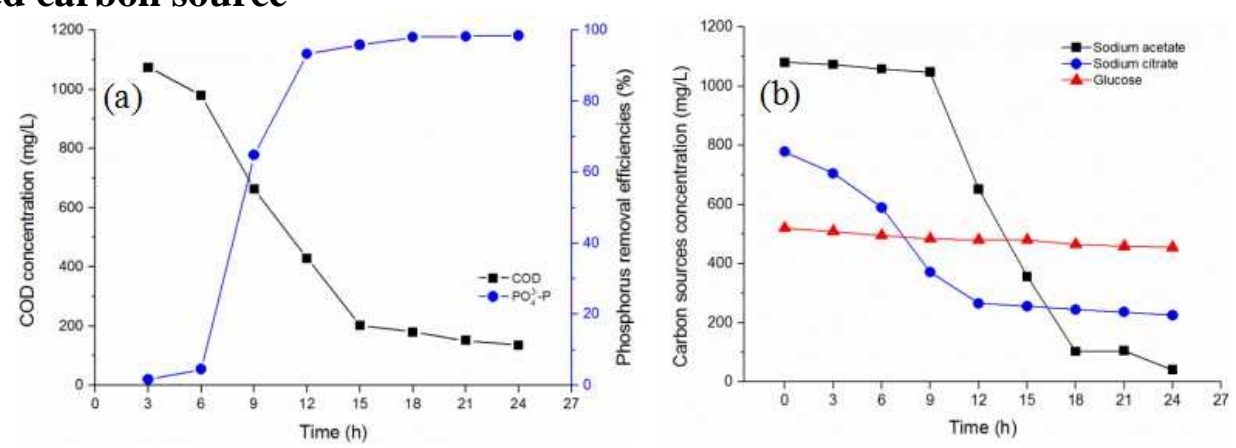

Fig.3 (a) Impact of mixed carbon source and (b) change of carbon sources concentration 
It could be seen from Fig. 3(a) that the $\mathrm{PO}_{4}{ }^{3-}-\mathrm{P}$ removal efficiency increased rapidly from $4.6 \%$ to $93.3 \%$ during $6 \mathrm{~h}-12 \mathrm{~h}$ and reached $98.45 \%$ eventually, while the increase in the others time was not obvious. The COD concentration showed corresponding decreasing trend in the meanwhile and dropped to $136 \mathrm{mg} / \mathrm{L}$ finally. More importantly, it could be intuitively seen from Fig. 3(b) that different carbon source presented various downtrends in the same system. Sodium acetate concentration dropped slowly in the first $9 \mathrm{~h}$, then it decreased relatively quickly until $18 \mathrm{~h}$, and it declined slowly to $0 \mathrm{mg} / \mathrm{L}$ in the last few hours. In contrast, concentration of sodium citrate decreased relatively quickly at the very start and maintained this trend in the former $12 \mathrm{~h}$, then decreased slowly until $0.2304 \mathrm{~g} / \mathrm{L}$ finally. In addition, the concentration of glucose almost did not change in the whole reaction time.

These changing trends were corresponded with that of Fig. 2(d), which further showed that the PK1 use carbon source selectively, taking priority using sodium citrate and basically not using glucose. For prokaryote, the utilization of sodium acetate was relatively complex. Firstly, sodium acetate should be catalyzed into acetyl-phosphate by acetokinase under the participation of adenosine triphosphate (ATP) and then transformed into acetyl-coA under the catalysis of phosphotransacetylase from Bacillus stearothermophilus, finally participated in the TAC. Therefore, compared with sodium citrate, which could participate in the TAC directly, strain PK1 would absorb sodium citrate rather than sodium acetate in the early stages [19]. Furthermore, the influence of glucose on phosphorus removal system was still a controversial issue. Some researches had proved that the phosphorus removal system would collapse with glucose as the sole carbon source, just as the results observed in this experiment. Because, glucose is a volatile fatty acids and its metabolic pathways are also very complex. But there were also a lot of experiments and mathematical models had proved that it could achieve good phosphorus removal effect with glucose in laboratory-scale reactor systems [20, 21].

\section{Conclusions}

(1) The increase of initial concentration of phosphorus could promote the growth PK1, but it would largely reduce the phosphorus removal efficiencies.

(2) PK1 showed different phosphorus removal efficiencies with different carbon sources. When sodium acetate, sodium citrate and mixed carbon source were used as the carbon sources, PK1 had high phosphorus removal efficiencies of $98.58 \%, 5.14 \%$ and $98.45 \%$ respectively.

(3) PK1 used carbon source selectively when using mixed carbon source, giving priority to utilize sodium citrate, then using sodium acetate, basically not using glucose.

\section{Acknowledgements}

This work was financially supported by the National Natural Science Foundation of China (NSFC) (NO. 51208397), and the Fundamental Research Funds for the Central Universities (NO. 2017II29GX).

\section{References}

[1] T. Mino, M. C. M. van Loosdrecht, and J.J. Heijnen: Water Res. Vol. 32 (1998), p. 3193

[2] H. Chen, D. Wang, X. Li, Q. Yang, K. Luo, and G. Zeng: Environ. Sci. Pollut. Res. Vol. 21 (2014), p. 6034

[3] M. Vargas, C. Casas, and J.A. Baeza: Biochem. Eng. J. Vol. 43 (2009), p. 288

[4] J. Guerrero, A. Guisasola, and J.A. Baeza: Water Res. Vol. 45 (2011), p. 4793

[5] X. Zheng, P. Sun, J. Han, Y. Song, Z. Hu, and H. Fan: Process Biochem. Vol. 49 (2014), p. 2207

[6] D. Jenkins, and V. Tandoi V: Water Res. Vol. 25 (1991), p. 1471 
[7] J.S. Jørgensen, and A.S.L. Pauli: Anaerobe Vol. 1 (1995), p. 161

[8] APHA: Standard methods for the examination of water and wastewater, 22nd ed. Denver: Amer Public Health Assn (2012)

[9] P. Canizares, A.D. Lucas, L. Rodriguez, and J. Villasenor: Environ. Technol. Vol. 21 (2000), p. 397

[10] I.M. Sudiana, T. Mino, H. Satoh, K. Nakamura, and T. Matsuo: Water Sci. Technol. Vol. 39 (1999), p. 29

[11] M. Okada, A. Murakami, C.K. Lin, and A. Murakami: Water Sci. Technol. Vol. 23 (1991), p. 755

[12] A. Carucci, K. Lindrea, M. Majone, and R. Ramadori: Water Sci. Technol. Vol. 39 (1999), p. 21

[13] J.S. Cech, and P. Hartman: Environ. Technol. Vol. 11 (1990), p. 651

[14] S.B. Noronha, H.J.C. Yeh, T.F. Spande, and J. Shiloach: Biotechnol. Bioeng. Vol. 68 (2000), p. 316

[15] S. Kumari, R. Tischel, M. Eisenbach, and A.J. Wolfe: J. Bacteriol. Vol. 177 (1995), p. 2868

[16] S. Kumari, C.M. Beatty, D.F. Browning, S.J. Busby, E.J. Simel, G. Hovel-Miner: J. Bacteriol. Vol. 182 (2000), p. 4173

[17] G. Gottschalk: Bacterial metabolism. New York: Spring-Verlag (1985)

[18] B.J. Eikmanns, D. Rittmann, and H. Sahm: J. Bacteriol. Vol. 177 (1995), p. 774

[19] S.R. Maloy, and W.D. Nunn: J. Bacteriol. Vol. 149 (1982), p. 173

[20] N. Wang, J. Peng, and G. Hill: Water Res. Vol. 36 (2002), p. 49

[21] Y. Liu, G. Xue, S. Yu, and F. Zhao: J. Environ. Sci. Vol. 18 (2006), p. 670 